\title{
Compact localized states and localization dynamics in the dice lattice
}

\author{
S. M. Zhang ๑ and L. Jin $\oplus^{*}$ \\ School of Physics, Nankai University, Tianjin 300071, China
}

(Received 5 March 2020; revised 12 July 2020; accepted 23 July 2020; published 10 August 2020)

\begin{abstract}
The dice lattice supports Aharonov-Bohm caging when all the energy bands are flat for the half-quantum magnetic flux enclosed in each plaquette of the lattice. We analytically investigate the eigenstates and discuss the localization dynamics. We find that arbitrary excitation is compactly confined within the excited-site-related snowflake structures of the dice lattice; as a consequence that the nonzero-energy flatband localizes in the single snowflake, whereas the zero-energy flatband localizes in three nearest snowflakes that are connected in the form of a trident star. The localization dynamics of an arbitrary excitation is grasped from two dynamical behaviors of single-site excitation. For the single-site excitation at the center of a snowflake, the excitation is localized in that snowflake; whereas for the single-site excitation at the branch site of a snowflake, the excitation is localized in the three snowflakes that the branch site belongs to. Our findings deepen the understanding of destructive interference and the dynamics of Aharonov-Bohm caging in the dice lattice.
\end{abstract}

DOI: 10.1103/PhysRevB.102.054301

\section{INTRODUCTION}

Flatbands are dispersiveless, fully constituted by degenerate energies and independent of the momentum [1]. The eigenstates of flatbands are known as compact localized states (CLSs) [2]. The initial excitations as the superpositions of CLSs are confined, the intensities of the excitation remain unchanged over time in the propagation, or in the time-evolution process. In the condensed-matter physics, optics, and quantum physics, the flatbands exist in various lattices of onedimension (1D) and two-dimension (2D) geometries. These lattices include the rhombic [3-12], sawtooth [13,14], crossstitch [15-17], dice [18-21], honeycomb [22,23], kagome [24-26], and pyrochlore lattices [27-29]. The formation of flatbands, the CLSs, the localization, the effects of disorder, as well as the interaction and nonlinearity are studied. In the experimental aspect, the Lieb lattice [30] possesses a zeroenergy flatband [31-36] and is realized in optical systems for cold atoms [26,37,38] and trapped ions [6]. It is also fabricated through the direct laser writing of optical waveguides [34-36]. Besides, an electronic Lieb lattice is designed [39]. The discovery of parity-time symmetry has triggered the exploration of non-Hermitian physics, including the flatband in the non-Hermitian optical metamaterials [40-47].

We emphasis that the flatband does not ubiquitously exist in physics. To generate the flatband in a system, there are several mechanisms where the destructive interference plays a pivotal role. For example, the chiral symmetry enables the

\footnotetext{
*jinliang@nankai.edu.cn
}

Published by the American Physical Society under the terms of the Creative Commons Attribution 4.0 International license. Further distribution of this work must maintain attribution to the author(s) and the published article's title, journal citation, and DOI. presence of a zero-energy flatband [48]. The symmetry ensures that the eigenstates compactly localize in one or several unit cells under the destructive interference. The localization dynamics is of vital importance for the flatband investigations. In the aforementioned systems possessing flatbands, most of them have only one flatband. Consequently, the CLSs in the flatband systems are the only excitations that can be completely confined; and this limits the many potential applications of the flatband. With the assistance of an effective magnetic field, the interference is tunable. When a half-quantum magnetic flux $\pi$ is introduced into a quasi-1D rhombic lattice, all the energy bands become flat [49]; and the excitations that are not limited to be the eigenstates of the system can be completely confined in certain regions, referred to as the Aharonov-Bohm (AB) caging. Recently, a rhombic optical realization of an $\mathrm{AB}$ cage was demonstrated experimentally in the coupled waveguide lattice with a $\pi$-synthetic magnetic flux engineered in each rhombic plaquette. The photonic caging of complete light confinement is demonstrated in the quasi-1D rhombic lattice [50,51]. In 2D, the dice lattice is one of the examples that has a spectrum fully constituted by the flatbands and supports the $\mathrm{AB}$ caging [18-20]; however, the CLSs of the dice lattice and the caging dynamics are not systematically explored. It is comparably more complicated to comprehend the 2D confinement mechanism. Nevertheless, understanding the localization in the dice lattice advantages the flatband physics in 2D, including the development on the flatband engineering, the quantum information processing, quantum computing, and the many other potential applications.

In this paper, we systematically demonstrate the properties of CLSs and the confinement dynamics of $\mathrm{AB}$ caging in the 2D dice lattice. We analytically investigate the configuration, the distribution, and the oscillation of the localization dynamics for an arbitrary excitation. The Peierls phases in the diamond plaquette of the dice lattice lead to the destructive 
interference as well as the flatbands. The eigenstates of the flatbands are analytically obtained. All the flatband eigenstates are the CLSs, and those corresponding to the same flatband are classified into two inequivalent configurations and related to the double degeneracy of the flatband. The CLSs with nonzero energies distribute in a single snowflake and are all linearly independent; whereas those with zeroenergy spread in a compound trident star constituted of three connected snowflakes. Notably, not all the zero-energy CLSs are linearly independent. The dice lattice supports the complete confinement of an arbitrary excitation. The excitations are well confined inside the regions that are covered by the snowflake structures of the related CLSs of the dice lattice. The $\mathrm{AB}$ caging of different types of initial excitations are thoroughly demonstrated in the numerical simulations.

The remainder of the paper is organized as follows. In Sec. II, the dice lattice is introduced; the spectrum with all bands flat is presented. In Sec. III, eigenstates of the dice lattice in the form of CLSs are analytically investigated; the orthogonality of the CLSs is discussed. In Sec. IV, the localization dynamics of the CLS excitations and several typical examples of excitations in the general form are performed and demonstrated. In Sec. V, results of the CLSs and the localization dynamics in the dice lattice are summarized.

\section{DICE LATTICE}

In this section, we introduce the dice lattice model. The dice lattice is also referred to as the $\mathcal{T}_{3}$ lattice [52-54]; the topological properties of which are extensively investigated in recent years [55-59], beyond the study of interaction in the dice lattice $[19,55,60,61]$. The dice lattice in the real space is schematically illustrated in Fig. 1. The dice lattice is a honeycomb lattice with an additional sublattice placed at the hexagonal center, and the additional sublattice is coupled to the three sublattices of the original honeycomb lattice [54]. The couplings of the dice lattice are uniform in contrast to the $\alpha-\mathcal{T}_{3}$ lattice with distinct couplings from the hexagonal vertexes to the hexagonal center [62-67]. The dice lattice under consideration shown in Fig. 1 is threaded by the half-quantum magnetic fluxes. The nonreciprocal coupling $i J$ along the direction of a black arrow has an $i=e^{i \pi / 2}$ Peierls phase factor in the front, and the corresponding coupling strength is $J$. The basic building block of the dice lattice includes two types of diamond plaquettes, the plaquette is $1 / 3$ of the hexagon as indicated in Fig. 1. Both types of plaquettes include four nonreciprocal couplings $i J$. Three of the nonreciprocal couplings are toward the identical direction of clockwise or counterclockwise; meanwhile, the other nonreciprocal coupling holds an opposite direction. Under the influence of the nonreciprocal coupling $i J$, each diamond plaquette of the dice lattice encloses a half-quantum magnetic flux $3 \pi / 2-\pi / 2=\pi$ or an equivalent one $-3 \pi / 2+\pi / 2=-\pi$. Notably, the diamond plaquette is the building block of the dice lattice but not the unit cell [16]. To compose the dice lattice in Fig. 1, the diamond plaquettes are arranged on the 2D plane under different orientations.

The dice lattice has been proposed in the optical lattice by using three pairs of counterpropagating linearly polarized laser beams of identical wavelength $\lambda=3 a_{0} / 2$

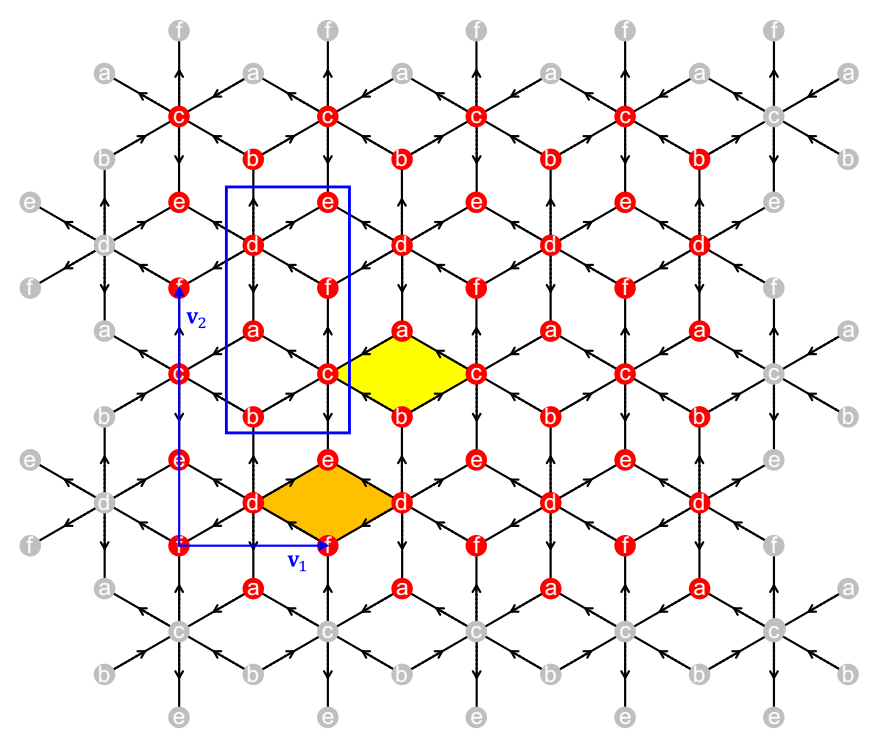

FIG. 1. Schematic of the 2D dice lattice. The uniform coupling has a nonreciprocal Peierls phase factor $e^{i \pi / 2}$ indicated by the arrow, being $i J$ for photons tunneling along the arrow. The diamond plaquette enclosed half-quantum magnetic flux $\pi$ is the building block of the dice lattice. The unit cell of the dice lattice involves six sites $a, b, c, d, e$, and $f$. The vectors $\mathbf{v}_{1}$ and $\mathbf{v}_{2}$ in the horizontal and the vertical directions are indicated by the blue arrows. The two types of diamond plaquettes are shaded in yellow and orange.

$[53,55,60,68]$. The laser beams are placed to divide the 2D plane into six equal sections, and $a_{0}$ is the lattice constant. The interference of the laser beams forms standing waves and generates the potentials for the optical lattice. The particles are confined at the potential minima. Considering the nearestneighbor couplings within a tight-binding model, the dice lattice captures the fundamental physics of particles in the optical lattice $[60,68]$. The optical lattice is controlled by the laser field intensities and phases. Tuning the phase of one pair of lasers induces the tunable magnetic flux enclosed in the plaquettes of the dice lattice. Alternatively, we point out that the dice lattice can be realized in the photonic crystal lattice of coupled resonators, which is an outstanding platform for the investigation of 2D lattices [69-72]. The proposed dice lattice is schematically shown in Fig. 2. Simply through introducing additional resonators in the hexagonal centers of the honeycomb lattice [71]. The ring-shape resonators are the primary resonators. The stadium-shape resonators are the linking resonators that are evanescently coupled with the primary resonators and induce the effective couplings between the primary resonators. The optical path-length difference experienced by the photons in the tunneling process between the primary resonators determines the Peierls phase factor in the effective couplings and induces the synthetic magnetic flux enclosed in the plaquettes. The synthetic magnetic flux is tunable through modifying the couplings between the linking resonators and the primary resonators [73].

The dice lattice is translational invariant along the horizontal direction for every another site and is translational invariant along the vertical direction for every four sites. The dice lattice is schematically illustrated in Fig. 1. The dice lattice 


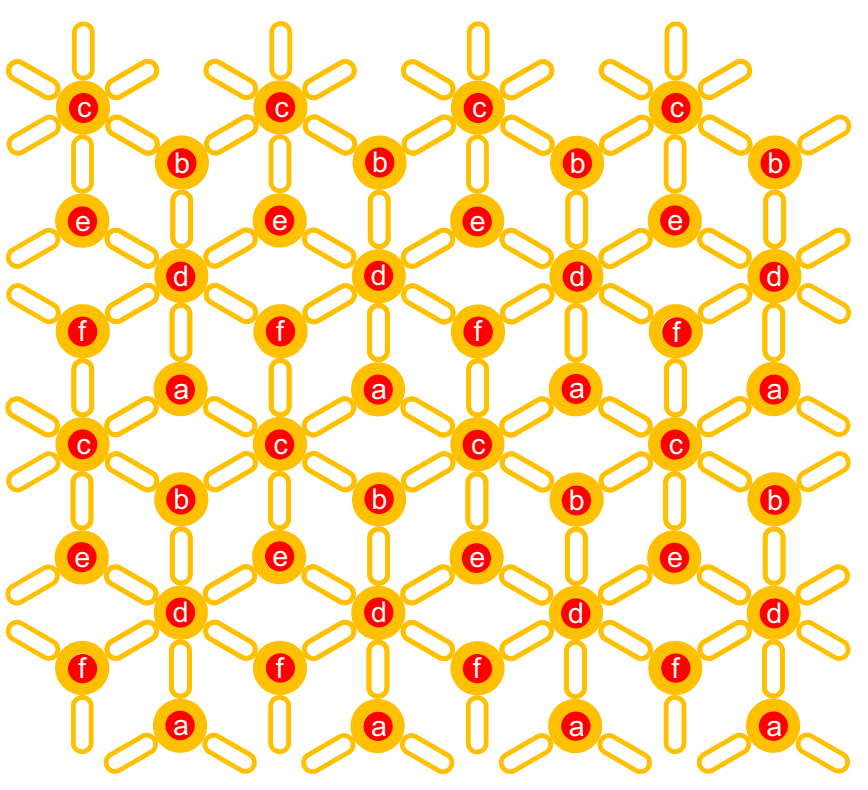

FIG. 2. Schematic of the 2D dice lattice of coupled resonators. The primary resonators are the ring-shape resonators marked with the sublattice types. The auxiliary resonators are the stadium-shape resonators in between the primary resonators. The total size of the dice lattice illustrated is $N=48$ with $m=4, n=12$.

Hamiltonian in the real space reads

$$
\begin{aligned}
H=i J \sum_{\mathbf{r}_{a}} & \left(a_{\mathbf{r}_{a}}^{\dagger} c_{\mathbf{r}_{c}}-a_{\mathbf{r}_{a}}^{\dagger} c_{\mathbf{r}_{c}-\mathbf{v}_{1}}-b_{\mathbf{r}_{b}}^{\dagger} c_{\mathbf{r}_{c}}-b_{\mathbf{r}_{b}}^{\dagger} c_{\mathbf{r}_{c}-\mathbf{v}_{1}}+a_{\mathbf{r}_{a}}^{\dagger} d_{\mathbf{r}_{d}}\right. \\
& +b_{\mathbf{r}_{b}}^{\dagger} d_{\mathbf{r}_{d}-\mathbf{v}_{2}}-c_{\mathbf{r}_{c}}^{\dagger} f_{\mathbf{r}_{f}}-c_{\mathbf{r}_{c}}^{\dagger} e_{\mathbf{r}_{e}-\mathbf{v}_{2}}-d_{\mathbf{r}_{d}}^{\dagger} e_{\mathbf{r}_{e}} \\
& \left.-d_{\mathbf{r}_{d}}^{\dagger} e_{\mathbf{r}_{e}-\mathbf{v}_{1}}+d_{\mathbf{r}_{d}}^{\dagger} f_{\mathbf{r}_{f}}-d_{\mathbf{r}_{d}}^{\dagger} f_{\mathbf{r}_{f}-\mathbf{v}_{1}}+\text { H.c. }\right)
\end{aligned}
$$

where the operators $O_{\mathbf{r}}^{\dagger}$ and $O_{\mathbf{r}}$ are the creation and annihilation operators for the six sublattices $O=$ $a, b, c, d, e$, and $f$ as indicated in a unit cell of the dice lattice in Fig. 1. The subscript $\mathbf{r}$ of the operators indicates the position in the dice lattice. For example, $\mathbf{r}_{a}$ is the position vector of the sublattice $a$ in a certain unit cell; and $\mathbf{r}_{b}=\mathbf{r}_{a}+$ $\left(0,-a_{0}\right), \mathbf{r}_{c}=\mathbf{r}_{a}+\left(\sqrt{3} a_{0} / 2,-a_{0} / 2\right), \mathbf{r}_{d}=\mathbf{r}_{a}+\left(0, a_{0}\right)$, $\mathbf{r}_{e}=\mathbf{r}_{a}+\left(\sqrt{3} a_{0} / 2,3 a_{0} / 2\right), \mathbf{r}_{f}=\mathbf{r}_{a}+\left(\sqrt{3} a_{0} / 2, a_{0} / 2\right)$ are the positions of the corresponding sublattices $b, c, d, e$, and $f$ in the same unit cell. The sublattices $a, b, e$, and $f$ are the rim sites of the dice lattice. The sublattices $c$ and $d$ are the hub sites of the dice lattice. The dice lattice is under periodic boundary conditions along both the horizontal and the vertical directions. The summation is all over the unit cells. The vectors $\mathbf{v}_{1}=\left(\sqrt{3} a_{0}, 0\right)$ and $\mathbf{v}_{2}=\left(0,3 a_{0}\right)$ describe the distance between two adjacent unit cells. We consider a general case; the site number in the horizontal (vertical) direction is $m(n)$; the total site number of the dice lattice under investigation is $N=m \times n$. For example, the dice lattice in Fig. 2 has $48=4 \times 12$ sites in total.

The Hamiltonian in the momentum space $H_{\mathbf{k}}$ is acquired through applying the Fourier transformations to the real-space dice lattice Hamiltonian $H$. Taking the Fourier transformations,

$$
\begin{array}{ll}
a_{\mathbf{r}_{a}}=\frac{1}{\sqrt{N}} \sum_{\mathbf{k}} e^{-i \mathbf{k} \cdot \mathbf{r}_{a}} a_{\mathbf{k}}, & b_{\mathbf{r}_{b}}=\frac{1}{\sqrt{N}} \sum_{\mathbf{k}} e^{-i \mathbf{k} \cdot \mathbf{r}_{b}} b_{\mathbf{k}}, \\
c_{\mathbf{r}_{c}}=\frac{1}{\sqrt{N}} \sum_{\mathbf{k}} e^{-i \mathbf{k} \cdot \mathbf{r}_{c}} c_{\mathbf{k}}, & d_{\mathbf{r}_{d}}=\frac{1}{\sqrt{N}} \sum_{\mathbf{k}} e^{-i \mathbf{k} \cdot \mathbf{r}_{d}} d_{\mathbf{k}}, \\
e_{\mathbf{r}_{e}}=\frac{1}{\sqrt{N}} \sum_{\mathbf{k}} e^{-i \mathbf{k} \cdot \mathbf{r}_{e}} e_{\mathbf{k}}, & f_{\mathbf{r}_{f}}=\frac{1}{\sqrt{N}} \sum_{\mathbf{k}} e^{-i \mathbf{k} \cdot \mathbf{r}_{f}} f_{\mathbf{k}},
\end{array}
$$

where $\mathbf{k}$ is the momentum in $2 \mathrm{D}$. The dice lattice has six sublattices; thus, we obtain a $6 \times 6$ matrix,

$$
H_{\mathbf{k}}=J\left(\begin{array}{cccccc}
0 & 0 & \Lambda_{k_{x}} & i & 0 & 0 \\
0 & 0 & \Gamma_{k_{x}} & i e^{i k_{y}} & 0 & 0 \\
\Lambda_{k_{x}}^{*} & \Gamma_{k_{x}}^{*} & 0 & 0 & -i e^{i k_{y}} & -i \\
-i & -i e^{-i k_{y}} & 0 & 0 & \Gamma_{k_{x}} & \Lambda_{k_{x}} \\
0 & 0 & i e^{-i k_{y}} & \Gamma_{k_{x}}^{*} & 0 & 0 \\
0 & 0 & i & \Lambda_{k_{x}}^{*} & 0 & 0
\end{array}\right)
$$

where $k_{x}=\mathbf{k} \cdot \mathbf{v}_{1}$ and $k_{y}=\mathbf{k} \cdot \mathbf{v}_{2}$ are the dimensionless momenta in the horizontal and vertical directions, respectively, and $k_{x}, k_{y} \in[-\pi, \pi]$. In $H_{\mathbf{k}}$, we have $\Lambda_{k_{x}}=2 e^{i k_{x} / 2} \sin \left(k_{x} / 2\right)$ and $\Gamma_{k_{x}}=-2 i e^{i k_{x} / 2} \cos \left(k_{x} / 2\right)$. The asterisk represents the complex conjugation operation. The six eigenenergies of $H_{\mathbf{k}}$ are doubly degenerate, forming three momentum independent flatbands with energies $\{-\sqrt{6} J, 0, \sqrt{6} J\}$ by solving the secular equation of $H_{\mathbf{k}}$. Enclosing a half-quantum magnetic flux in each plaquette of the 2D dice lattice induces a completely flat spectrum, which leads to an $\mathrm{AB}$ cage manipulated by the destructive interference.

\section{COMPACT LOCALIZED STATES}

The dice lattice spectrum is fully flat as a consequence of the destructive interference, caused by the interplay between nonreciprocal couplings $\pm i J$; in this situation, the eigenstates of the dice lattice are CLSs. In this section, we analytically discuss the eigenstates of the dice lattice from the view point of destructive interference, that is, the cancellation of the wave-function superposition associated with the couplings. This will deepen our understanding of the flatband and the localization effect in the dice lattice.

The 2D lattice is more complicated than the $1 \mathrm{D}$ lattice. The configuration of CLSs may not be coincident with the configuration of the unit cells. With different types and orientations of the diamond plaquettes in the dice lattice, there are many different ways to choose the unit cell. These hinder uncovering the localization properties of the dice lattice. To understand the dice lattice, we emphasize the importance of the snowflake structures. The snowflake configuration includes six branch sites that are coupled to the central site as indicated in Fig. 1. The identical snowflakes are connected one by one in the horizontal direction; two configurations of horizontally coupled snowflakes are connected and alternatively present in the vertical direction. Notably, each snowflake connects to its six adjacent snowflakes through sharing two sites. The two types of snowflakes can also be regarded as the building blocks of the dice lattice; in this viewpoint, the configurations of CLSs 
in the dice lattice are straightforwardly understood from the destructive interference, and the wave-function amplitudes of CLSs are easily obtained.

In the dice lattice, the repetition manner of the snowflakes is identical to the repetition manner of the unit cells. Both two types of snowflakes have two nonzero-energy eigenstates and five degenerate zero-energy eigenstates, being identical to the three flatband energies of the dice lattice. The CLSs of the flatband, instead of being localized in the configurations formed by the unit cells, are compactly localized in the configurations of snowflakes.

The matrix form Hamiltonian of the type-I snowflake is

$$
h_{\mathrm{I}}=\left(\begin{array}{ccccccc}
0 & 0 & 0 & i J & 0 & 0 & 0 \\
0 & 0 & 0 & i J & 0 & 0 & 0 \\
0 & 0 & 0 & i J & 0 & 0 & 0 \\
-i J & -i J & -i J & 0 & -i J & -i J & i J \\
0 & 0 & 0 & i J & 0 & 0 & 0 \\
0 & 0 & 0 & i J & 0 & 0 & 0 \\
0 & 0 & 0 & -i J & 0 & 0 & 0
\end{array}\right) .
$$

The steady-state Schrödinger equations for $h_{\mathrm{I}}$ are

$$
h_{\mathrm{I}}\left|\psi_{\mathrm{I}}\right\rangle=E\left|\psi_{\mathrm{I}}\right\rangle
$$

The energies of $h_{\mathrm{I}}$ satisfy $\operatorname{det}\left(h_{\mathrm{I}}-E I_{7}\right)=0$, where $I_{7}$ represents the $7 \times 7$ identity matrix. Thus, the energies of $h_{\mathrm{I}}$ can be obtained from $E^{5}\left(6 J^{2}-E^{2}\right)=0$. The corresponding eigenstates are obtained from the steady-state Schrödinger equations,

$$
\begin{aligned}
i J \psi_{4} & =E \psi_{1}, \\
i J \psi_{4} & =E \psi_{2}, \\
i J \psi_{4} & =E \psi_{3}, \\
-i J\left(\psi_{1}+\psi_{2}+\psi_{3}+\psi_{5}+\psi_{6}\right)+i J \psi_{7} & =E \psi_{4}, \\
i J \psi_{4} & =E \psi_{5}, \\
i J \psi_{4} & =E \psi_{6}, \\
-i J \psi_{4} & =E \psi_{7} .
\end{aligned}
$$

The unnormalized eigenstate for the nonzero-energy $\pm \sqrt{6} J$ is

$$
\left|\psi_{\mathrm{I}}\right\rangle=[-1,-1,-1, \pm i \sqrt{6},-1,-1,1]^{T},
$$

which is the type-I CLSs of the dice lattice illustrated in Fig. 3 on the top left. The five unnormalized linearly independent degenerate zero-energy eigenstates are

$$
\left|\psi_{\mathrm{I}}\right\rangle=\left(\begin{array}{c}
1 \\
-1 \\
0 \\
0 \\
0 \\
0 \\
0
\end{array}\right),\left(\begin{array}{c}
1 \\
0 \\
-1 \\
0 \\
0 \\
0 \\
0
\end{array}\right),\left(\begin{array}{c}
1 \\
0 \\
0 \\
0 \\
-1 \\
0 \\
0
\end{array}\right),\left(\begin{array}{c}
1 \\
0 \\
0 \\
0 \\
0 \\
-1 \\
0
\end{array}\right),\left(\begin{array}{l}
1 \\
0 \\
0 \\
0 \\
0 \\
0 \\
1
\end{array}\right) .
$$

Type-I
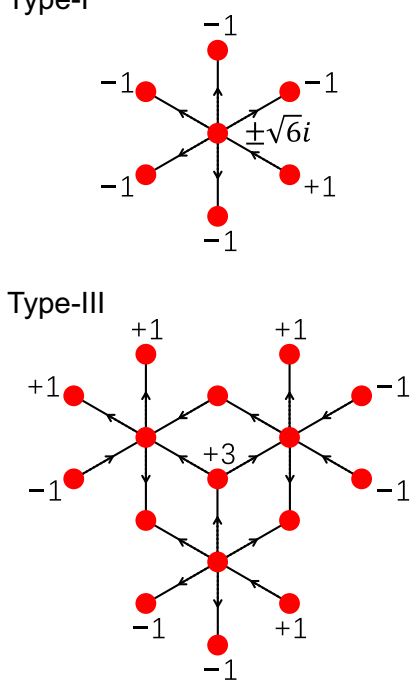

Type-IV

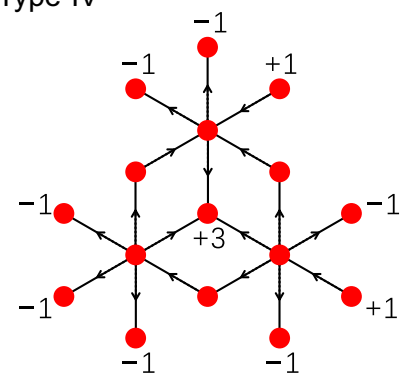

Type-V
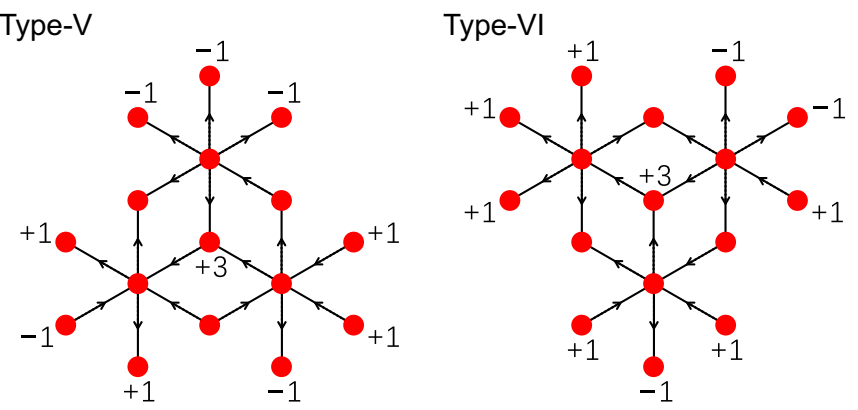

FIG. 3. Schematic of the six types of CLSs of the dice lattice. The energies of the CLSs are $\pm \sqrt{6} J$ for the top panel and 0 for the middle and bottom panels. The wave-function values of the unmarked sites are zero.

The situation for the snowflake of type-II is similar, the matrix form Hamiltonian of the type-II snowflake reads

$$
h_{\mathrm{II}}=\left(\begin{array}{ccccccc}
0 & 0 & 0 & i J & 0 & 0 & 0 \\
0 & 0 & 0 & -i J & 0 & 0 & 0 \\
0 & 0 & 0 & i J & 0 & 0 & 0 \\
-i J & i J & -i J & 0 & -i J & i J & i J \\
0 & 0 & 0 & i J & 0 & 0 & 0 \\
0 & 0 & 0 & -i J & 0 & 0 & 0 \\
0 & 0 & 0 & -i J & 0 & 0 & 0
\end{array}\right) .
$$

From the steady-state Schrödinger equations $h_{\mathrm{II}}\left|\psi_{\mathrm{II}}\right\rangle=E\left|\psi_{\mathrm{II}}\right\rangle$, we obtain the unnormalized eigenstate for the nonzero-energy $\pm \sqrt{6} J$ as

$$
\left|\psi_{\mathrm{II}}\right\rangle=[-1,1,-1, \pm i \sqrt{6},-1,1,1]^{T},
$$

which is the type-II CLSs of the dice lattice illustrated in Fig. 3; the other five unnormalized linearly independent 
degenerate zero-energy eigenstates are

$$
\left|\psi_{\mathrm{II}}\right\rangle=\left(\begin{array}{l}
1 \\
1 \\
0 \\
0 \\
0 \\
0 \\
0
\end{array}\right),\left(\begin{array}{c}
1 \\
0 \\
-1 \\
0 \\
0 \\
0 \\
0
\end{array}\right),\left(\begin{array}{c}
1 \\
0 \\
0 \\
0 \\
-1 \\
0 \\
0
\end{array}\right),\left(\begin{array}{l}
1 \\
0 \\
0 \\
0 \\
0 \\
1 \\
0
\end{array}\right),\left(\begin{array}{l}
1 \\
0 \\
0 \\
0 \\
0 \\
0 \\
1
\end{array}\right) .
$$

Any superposition of the degenerate zero-energy eigenstates is still the eigenstate. We point out that the key feature of the zero-energy eigenstates for the Hamiltonian of the snowflake is the vanishing amplitude on its central site $\psi_{4}=0$ as a consequence of the destructive interference of the wave functions of the six branch sites. The zero-energy CLSs of the dice lattice are superpositions of these zero-energy eigenstates.

All the possible CLSs in the dice lattice are classified into six types of configurations according to the two inequivalent snowflake structures as well as the three flatband energies. In Fig. 3, the wave function of the CLSs in the real space are schematically illustrated; all the six types of CLS configurations of wave-function distributions are presented with their wave functions marked. The CLSs are all localized in the snowflakes. Apparently, each CLS configuration is inequivalent to the other five CLS configurations; however, all the possible CLSs in the form of the six CLS configurations found in the dice lattice are not necessarily orthogonal to each other and are not fully linear independent due to the doubly degeneracy of the flatbands. The CLSs of type-I and type-II shown in the top panel of Fig. 3 distribute in a single snowflake structure and are for the flatbands with nonzero energies $\sqrt{6} J$ and $-\sqrt{6} J$, with the value of the wave function at the center of the snowflake being $\sqrt{6} i$ and $-\sqrt{6} i$, respectively. For the zero-energy flatband, the CLSs from type-III to type-VI shown in the middle and bottom panels of Fig. 3 are distributed in three snowflakes that are connected in the form of a trident star.

It is straightforward to verify that the CLSs illustrated in Fig. 3 satisfy the series of discrete Schrödinger equations,

$$
H\left|\psi_{E}\right\rangle=E\left|\psi_{E}\right\rangle,
$$

in the whole 2D lattice plane, where $\left|\psi_{E}\right\rangle$ represents the wave function for the eigenvalue $E$. To be precise, the Schrödinger equations are a set of equations with their left sides being the summation of the products of the couplings and the related wave-function amplitudes; whereas the right sides of the Schrödinger equations are the product of the eigenvalue and the corresponding wave function. For the CLSs of both type-I and type-II, they satisfy the Schrödinger equations and can be verified as follows. The left side of the Schrödinger equation for the center site of the snowflake is the summation of six products of the branch couplings and the corresponding wave-function values, that is

$$
\begin{aligned}
& 5(-i J)(-1)+(i J)(+1)=6 i J, \\
& 3(-i J)(-1)+3(i J)(+1)=6 i J
\end{aligned}
$$

for type-I and type-II, respectively; the right side of the Schrödinger equation for the center site of the snowflake is the product of the flatband energy and the wave-function value at the center site, that is

$$
( \pm \sqrt{6} J)( \pm \sqrt{6} i)=6 i J
$$

and equals to the left side of the Schrödinger equation. The left side of the Schrödinger equation for the branch site of the snowflake is the product of the branch coupling and the wave-function value at the center site of the snowflake, that is

$$
\begin{aligned}
& (i J)( \pm \sqrt{6} i)=\mp \sqrt{6} J \\
& (-i J)( \pm \sqrt{6} i)= \pm \sqrt{6} J
\end{aligned}
$$

for the branch site with wave-function value $(-1)$ and $(+1)$, respectively; the right side of the Schrödinger equation for the branch site of the snowflake is the product of the flatband energy and the wave-function value at the branch site, that is

$$
\begin{aligned}
& ( \pm \sqrt{6} J)(-1)=\mp \sqrt{6} J \\
& ( \pm \sqrt{6} J)(+1)= \pm \sqrt{6} J
\end{aligned}
$$

for the branch site with wave-function values $(-1)$ and $(+1)$, respectively; thus, the left and right sides of the Schrödinger equations for the branch sites of the snowflake are also equal. These are in accord with the discussed nonzero eigenstate solutions of $h_{\mathrm{I}, \mathrm{II}}$.

The other four types of configurations from type-III to type-VI represent the zero-energy CLSs. The Schrödinger equations for the CLSs in these configurations can be verified after checking the validity of the Schrödinger equations for the 16 sites of the three snowflakes. Note that the corresponding flatband energy is zero; thus, the right side of the Schrödinger equation for every site is zero because it is the product of the flatband energy and the wave-function value. Besides, the wave-function values are zero for all the sites that connect to the center with wave-function value $(+3)$ in these configurations; thus, the left side of the Schrödinger equations for these 13 sites (except for the three snowflake centers) are all zero because they are all equal to the product of the zero wavefunction values and the corresponding couplings. Thus, the left and right sides of the Schrödinger equations for these 13 sites are all zero and being equal. We also need to calculate the left side of the Schrödinger equations for the three snowflake centers in the four configurations from type-III to type-IV. Let us take type-III as an elucidation, the summation of products of four nonvanishing wave-function values and the corresponding branch couplings $( \pm i J)$ for the snowflake on the top left of the configuration type-III is $(-1)(i J)+(+1)(-i J)+$ $(+1)(-i J)+(+3)(i J)=0$. Similarly, the left sides of the Schrödinger equations for the other snowflake centers in typeIII and all the others in type-IV, type-V, and type-VI are all zero. Thus, the CLSs in all the six configurations shown in Fig. 3 satisfy the Schrödinger equations of the dice lattice.

The CLSs of the dice lattice are completely localized because the CLSs are isolated snowflake configurations. This is because the wave functions at the two shared branch sites destructively interfere at the central site of each adjacent snowflake that connects to them. For type-I and type-II CLSs, there are six adjacent snowflakes connected with the CLSs configuration. For the type-III to type-VI CLSs, there are nine neighbor snowflakes connected with the CLSs configuration. 
The CLSs from different flatbands have different energies and are orthogonal to each other. Moreover, the degenerate CLSs of type-I and type-II from the same flatband with identical energy are still orthogonal to each other. Thus, all the CLSs of type-I and type-II are orthogonal and linearly independent, regardless of their energies. The eigenstates of the zero-energy flatband are CLSs from type-III to type-VI, but not all of them supported in the dice lattice are linearly independent as further explained in the Appendix.

The dice lattice shown in Fig. 1 is considered under periodic boundary conditions along both the horizontal and the vertical directions. The dice lattice has 48 sites in total; thus, there are 48 linearly independent CLS eigenstates. Note that the snowflake structures are distributed into four horizontal lines of the dice lattice, each horizontal line has four snowflakes. There are 16 snowflakes in total including 8 type-I snowflakes and 8 type-II snowflakes. Every snowflake supports two independent CLSs with energies $-\sqrt{6} J$ and $\sqrt{6} J$. Thus, there are 16 CLSs with energies $-\sqrt{6} J$ and 16 CLSs with energy $\sqrt{6} J$. All the 32 CLSs of type-I and type-II localized in the single snowflake are linearly independent. The rest 16 energy levels of the dice lattice belong to the zero-energy flatband. To summarize, the 48 size dice lattice has three flatbands with energy $-\sqrt{6} J, 0$, and $+\sqrt{6} J$; each flatband is constituted by 16 energy levels.

The eigenstates of the zero-energy flatband are CLSs of type-III to type-VI. Both the first and the third horizontal lines from the top of the dice lattice with four snowflakes support four type-III and four type-V zero-energy CLSs; both the second and the fourth horizontal lines from the top of the dice lattice with four snowflakes support four type-IV and four type-VI zero-energy CLSs. There are, in total, 32 CLSs of type-III to type-VI; however, all the CLSs of type-III to type-VI are not fully linearly independent, and only part of them are linearly independent. The fact is noted from the zero and nonzero overlaps between the CLSs of type-III to type-VI.

The time-evolution state of the initial excitation being the CLS is shown in Fig. 4. Figures 4(a)-4(d) are for the CLSs of flatbands with nonzero energies. Figures 4(e)-4(h) are for the CLSs of flatband with zero energy. The distribution of the excitation is well localized at the long time interval. With the analytical CLSs of all the flatbands, we can systematically investigate the $2 \mathrm{D}$ localization dynamics of $\mathrm{AB}$ caging in the dice lattice.

\section{LOCALIZATION DYNAMICS}

The complete confinement in a lattice with its spectrum full of flatbands is referred to as the $\mathrm{AB}$ caging [49-51]. We present the complete localization dynamics of the $\mathrm{AB}$ caging in the dice lattice on the premise of analytical CLSs. In this section, we demonstrate the time-evolution dynamics of the CLSs and the other states that are not the eigenstates of the dice lattice where the localization and fidelity in the timeevolution process are highlighted. Nondiffractive dynamics of all the eight CLSs are presented in Fig. 4; two types of representative single-site excitation and the time evolutions for the arbitrary initial states are demonstrated.

In Fig. 4, the CLS configuration is taken as the initial excitation $\Psi(0)$, and the numerical simulation of the time-evolved a)

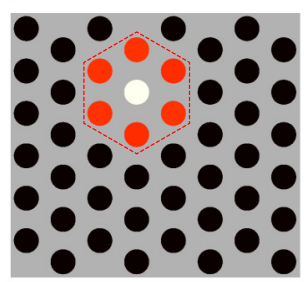

c)

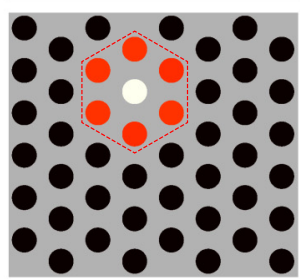

e)

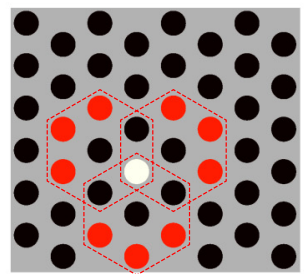

g)

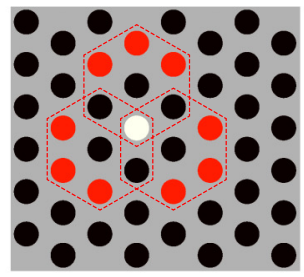

(b)

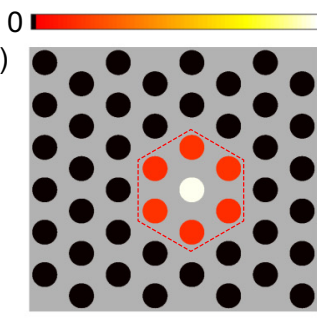

(d)

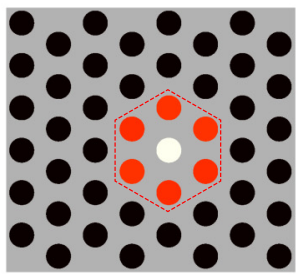

(f)

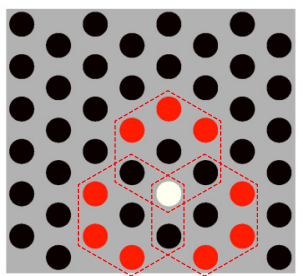

(h)



FIG. 4. Localization of normalized CLSs in the 48-site dice lattice under periodic boundaries. The intensity of excitation $\langle\Psi(t) \mid \Psi(t)\rangle$ of the evolved initial excitation is at a long time moment $t=100 / J$. The initial excitation is (a) the type-I CLS with energy $-\sqrt{6} J$, (b) the type-II CLS with energy $-\sqrt{6} J$, (c) the type-I CLS with energy $\sqrt{6} J$, (d) the type-II CLS with energy $\sqrt{6} J$, (e) the type-III CLS, (f) the type-IV CLS, (g) the type-V CLS, and (h) the type-VI CLS. The coupling is set to be unity $J=1$. The schematic of the dice lattice is in Fig. 1.

state $|\Psi(t)\rangle$ is exhibited for all the configurations of CLSs shown in Fig. 3. For the excitation being a single snowflake localized eigenstate, the intensity of excitation $\langle\Psi(t) \mid \Psi(t)\rangle$ is localized in the single snowflake without escaping; for the excitation being a zero-energy eigenstate, the intensity is completely localized within the three snowflakes without escaping. It is noted that the CLS distribution is well localized without changing; this exhibits the nondiffractive dynamics of the CLSs. Moreover, the time evolution of the superposition of CLSs is performed as follows. The dynamics is still nondiffractive and localized; and the excitation is confined within the areas where the CLSs have nonzero intensity distribution. Note that the eigenstates of the dice lattice under half-quantum magnetic flux are all CLSs. Any initial excitation in the dice lattice is a superposition of different configurations of CLSs; thus, the excitation in the dice lattice will be fully confined without escaping. From the localized CLSs of the dice lattice, it is expected that any form of the initial excitation must be confined in the compound snowflake structure as the superposition of the snowflakes of the related CLSs. In practice, the 
(a)

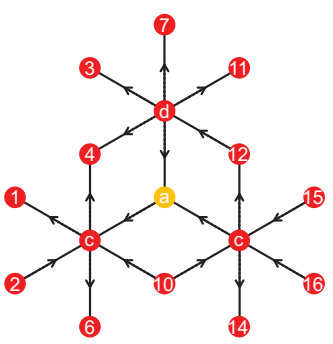

(c)

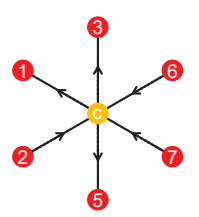

(e)

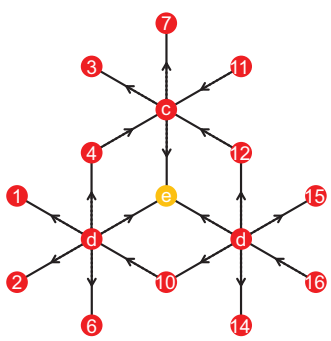

(b)

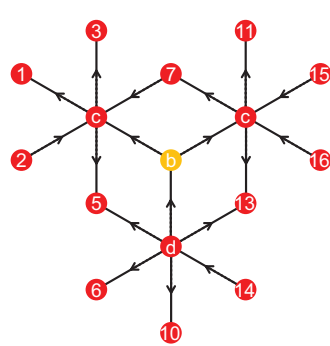

(d)

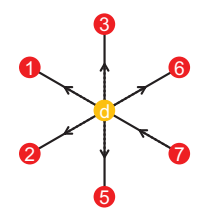

(f)

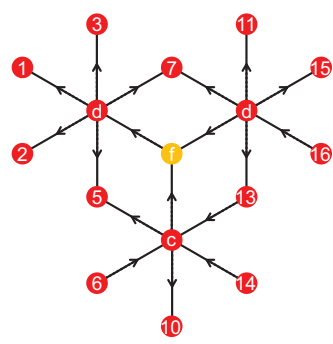

FIG. 5. Schematics of the single-site excitation (shaded in orange) on the sublattice sites from $a$ to $f$.

localization effect of the time-evolution dynamics in the dice lattice can be understood from the dynamics of the single-site excitation.

In the following, we systematically investigate the dynamics of an arbitrary excitation in the dice lattice. The dice lattice enclosed with half-quantum magnetic flux has six types of sublattices, including the sublattices $c, d$ positioned at the snowflake centers (hub sites) and the sublattices $a, b, e$, and $f$ positioned at the snowflake branch sites (rim sites) in the dice lattice. The analytical dynamics for an arbitrary initial state $\Psi(t)$ is the superposition of the evolved states of CLSs; that is,

$$
|\Psi(t)\rangle=e^{-i H t}|\Psi(0)\rangle=\sum_{j} \lambda_{j} e^{-i E_{j} t}\left|\psi_{j}\right\rangle,
$$

where $\left|\psi_{j}\right\rangle$ is the CLS with flatband energy $E_{j}$ and $\lambda_{j}$ is the superposition coefficient.

To calculate the time-evolution dynamics, we point out that any initial excitation is a superposition of the single-site excitation of the six types of sublattices. Thus, we first analytically calculate the dynamics of the six types of single-site excitation from which we, then, obtain the dynamics of an arbitrary excitation as a superposition of different single-site excitations. The single-site excitations on the sublattices $a, b, c, d, e$, and $f$ sites are illustrated in Fig. 5, respectively. The excitation will be confined in the related snowflake configurations.

For the excitation being the site $a$ that is shaded in orange in Fig. 5(a), the initial excitation is related to the CLSs of all three flatbands of the dice lattice. This form of the initial excitation is related to three snowflakes that support the independent CLSs of both flatbands with energies $-\sqrt{6} J$ and $\sqrt{6} \mathrm{~J}$. It is also related to the type-V CLS of the zeroenergy band centered at the initial excitation. Note that all the zero-energy CLSs have vanishing amplitudes at the three rim sites in the hexagonal structure except for the hexagonal center as elaborated in Fig. 3; thus, the initial excitation is not relevant to any other zero-energy CLSs, and the excitation is completely confined inside the three-snowflake area of the type-V zero-energy CLSs. The initial-state $\left|\Psi_{a}(0)\right\rangle$ is a superposition of seven CLSs including a zero-energy type-V CLS centered at site $a$, and three pairs of nonzero-energy CLSs centered at the three snowflake centers of site $d$ and two sites $c$, respectively; each pair of nonzero-energy CLSs have both energies $-\sqrt{6} J$ and $\sqrt{6} J$. Two type-I CLSs with the identical superposition coefficients $-1 / 12$ are required to obtain the vanishing distribution at site $d$ in the top snowflake for the initial-state $\left|\Psi_{a}(0)\right\rangle$. For the vanishing distribution of $\left|\Psi_{a}(0)\right\rangle$ at the two sites $c$ at the bottom left and the bottom right snowflake centers, the superposition coefficients for the two type-II CLSs are $1 / 12$ and $-1 / 12$, respectively. The superposition coefficient for the type-V CLS is $1 / 6$. The timeevolution state $\left|\Psi_{a}(t)\right\rangle$ can be written as

$$
\begin{aligned}
\left|\Psi_{a}(t)\right\rangle= & \frac{1}{6}\left|\psi_{\mathrm{V}, 0, a}\right\rangle-\frac{1}{12} \sum_{\sigma= \pm} e^{-i \sigma \sqrt{6} J t}\left(\left|\psi_{\mathrm{I}, \sigma, d}\right\rangle\right. \\
& \left.-\left|\psi_{\mathrm{II}, \sigma, c_{l}}\right\rangle+\left|\psi_{\mathrm{II}, \sigma, c_{r}}\right\rangle\right) \\
= & \frac{1}{6}[1-\beta, \beta-1, \beta-1,0, \sqrt{6} \alpha, 1-\beta, \beta-1 \\
& -\sqrt{6} \alpha, 3(\beta+1), 0, \beta-1,0,-\sqrt{6} \alpha \\
& \quad \beta-1,1-\beta, 1-\beta]^{T}
\end{aligned}
$$

where we set $\alpha=\sin (\sqrt{6} J t), \beta=\cos (\sqrt{6} J t)$. The Roman numerals in the subscripts indicate the types of the CLSs; the sign $\sigma$ indicates the corresponding nonzero flatband energy $\sigma \sqrt{6} J$; the letters $d, c_{l}$, and $c_{r}$ indicate where the CLSs centered, and the subscripts $l$ and $r$ describe the snowflake centers $c$ on the left side and on the right side, respectively. The numbers in the schematics in Fig. 5 label the index for the wave functions of $\left|\Psi_{a}(t)\right\rangle$. The fidelity as the overlap between the initial state and the time-evolution state is

$$
F_{a}(t)=\left|\left\langle\Psi_{a}(0) \mid \Psi_{a}(t)\right\rangle\right|=\frac{1}{2}(\beta+1) .
$$

For the single-site excitation at site $b$ as shown in Fig. 5(b), initial-state $\left|\Psi_{b}(0)\right\rangle$ is a superposition of seven CLSs with a type-III CLS centered at site $b$ and three pairs of nonzeroenergy CLSs localized at the snowflake centers. The timeevolution state $\left|\Psi_{b}(t)\right\rangle$ is

$$
\begin{aligned}
\left|\Psi_{b}(t)\right\rangle= & \frac{1}{6}\left|\psi_{\mathrm{III}, 0, b}\right\rangle+\frac{1}{12} \sum_{\sigma= \pm} e^{-i \sigma \sqrt{6} J t}\left(\left|\psi_{\mathrm{II}, \sigma, c_{l}}\right\rangle\right. \\
& \left.+\left|\psi_{\mathrm{II}, \sigma, c_{r}}\right\rangle-\left|\psi_{\mathrm{I}, \sigma, d}\right\rangle\right) \\
= & \frac{1}{6}[1-\beta, \beta-1,1-\beta, \sqrt{6} \alpha, 0, \beta-1,0, \\
& 3(\beta+1),-\sqrt{6} \alpha, \beta-1,1-\beta, \sqrt{6} \alpha, \\
& \quad 0,1-\beta, \beta-1, \beta-1]^{T} .
\end{aligned}
$$

The numbers in the schematics in Fig. 5 label the index for the wave functions of $\Psi_{b}(t)$. The fidelity as the overlap between 

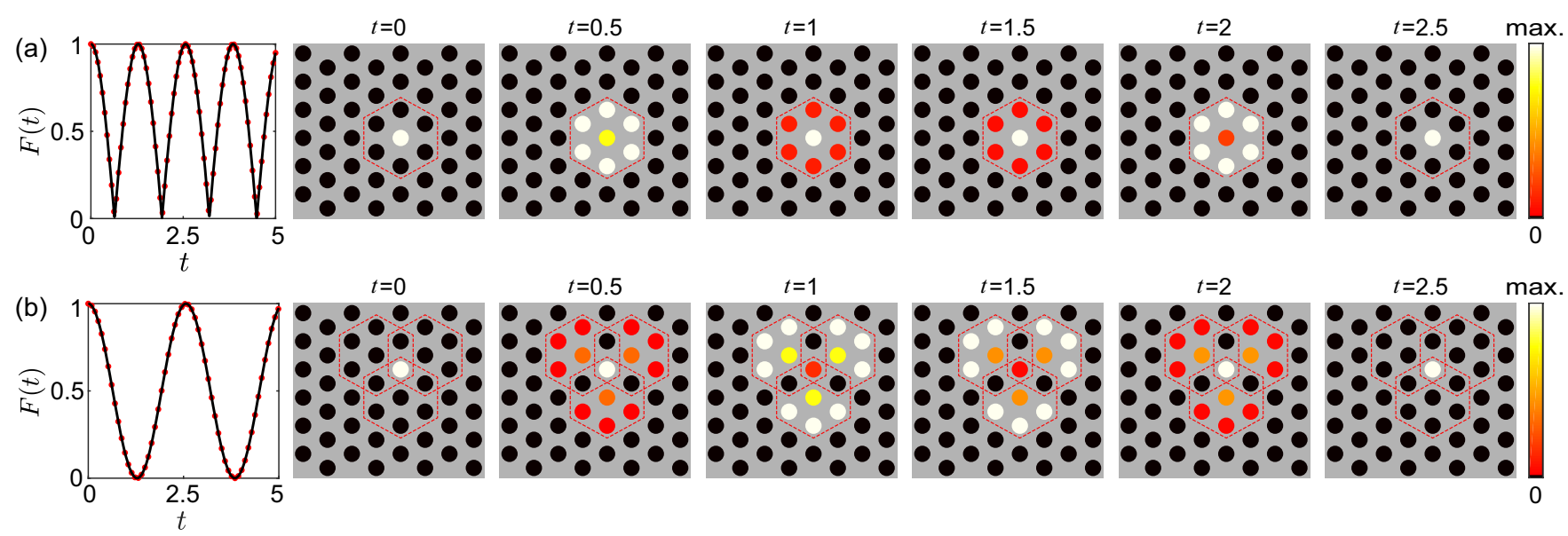

FIG. 6. Fidelity $F(t)=|\langle\Psi(0) \mid \Psi(t)\rangle|$ and snapshots of the intensity of excitation $\langle\Psi(t) \mid \Psi(t)\rangle$ in the time-evolution process for the singlesite initial excitation in the 48 -site dice lattice under periodic boundaries. The initial state is normalized, $\langle\Psi(0) \mid \Psi(0)\rangle=1$. The analytical results of $F(t)$ are indicated by the black solid lines; the numerical results of $F(t)$ are in the red dots. (a) The initial excitation is a single-site excitation at the center of the snowflake $\Psi_{c}(t)$. (b) The initial excitation is a single-site excitation at the branch site of the snowflake $\Psi_{f}(t)$. The schematic of the dice lattice is in Fig. 1.

the initial state and the time-evolution state is

$$
F_{b}(t)=\left|\left\langle\Psi_{b}(0) \mid \Psi_{b}(t)\right\rangle\right|=\frac{1}{2}(\beta+1) .
$$

Figure 6(a) performs the numerical simulation of the time evolution for a single-site excitation on a center site of the snowflake $\left|\Psi_{c}(t)\right\rangle$. In this situation, the initial excitation is unrelated to the CLSs of the zero-energy flatbands. This is because that the wave function at the snowflake center is zero for all the CLSs of zero-energy flatbands, which can be seen from the CLS distributions from type-III to type-VI shown in Fig. 3. Thus, the single-site excitation at the center of the snowflake only relates to the four CLSs with energies $-\sqrt{6} J$ and $\sqrt{6} J$; the type of CLSs is either type-I or type-II and depends on the snowflake being excited. Thus, the initial excitation is a superposition of such two kinds of nonzero CLSs localized in a single snowflake, and the excitation is completely confined inside the snowflake that the single-site initial state is excited. For the single-site excitation at the site $c$ as shown in Fig. 5(c), the initial state $\left|\Psi_{c}(0)\right\rangle$ is confined in the snowflake, being a superposition of the two nonzero-energy type-II CLSs, the superposition coefficient for the state with energy $\sqrt{6} J$ is $-\sqrt{6} i / 12$ and for state with energy $-\sqrt{6} J$ is $\sqrt{6} i / 12$. Therefore, the time-evolution state $\left|\Psi_{c}(t)\right\rangle$ is

$$
\begin{aligned}
\left|\Psi_{c}(t)\right\rangle & =-\frac{\sqrt{6} i}{12} \sum_{\sigma= \pm} \sigma e^{-i \sigma \sqrt{6} J t}\left|\psi_{\mathrm{II}, \sigma, c}\right\rangle \\
& =\frac{\sqrt{6}}{6}[\alpha,-\alpha, \alpha, \sqrt{6} \beta, \alpha,-\alpha,-\alpha]^{T} .
\end{aligned}
$$

The fidelity as the overlap between the initial state and the time-evolution state is

$$
F_{c}(t)=\left|\left\langle\Psi_{c}(0) \mid \Psi_{c}(t)\right\rangle\right|=|\beta| .
$$

For the single-site excitation at site $d$ as shown in Fig. 5(d), initial state $\left|\Psi_{d}(0)\right\rangle$ is a superposition of the two nonzero-energy type-I CLSs; the superposition coefficients are $-\sqrt{6} i / 12$ for the CLS with energy $\sqrt{6} J$ and $\sqrt{6} i / 12$ for the CLS with energy $-\sqrt{6} J$. Therefore, the time-evolution state $\left|\Psi_{d}(t)\right\rangle$ is

$$
\begin{aligned}
\left|\Psi_{d}(t)\right\rangle & =-\frac{\sqrt{6} i}{12} \sum_{\sigma= \pm} \sigma e^{-i \sigma \sqrt{6} J t}\left|\psi_{\mathrm{I}, \sigma, d}\right\rangle \\
& =\frac{\sqrt{6}}{6}[\alpha, \alpha, \alpha, \sqrt{6} \beta, \alpha, \alpha,-\alpha]^{T} .
\end{aligned}
$$

The fidelity as the overlap between the initial state and the time-evolution state is

$$
F_{d}(t)=\left|\left\langle\Psi_{d}(0) \mid \Psi_{d}(t)\right\rangle\right|=|\beta| .
$$

For the single-site excitation at the site $e$ as shown in Fig. 5(e), initial-state $\left|\Psi_{e}(0)\right\rangle$ is a superposition of seven CLSs with a type-IV CLS centered at site $e$ and three pairs of nonzero-energy CLSs localized at the snowflake centers. The time-evolution state $\left|\Psi_{e}(t)\right\rangle$ is

$$
\begin{aligned}
\left|\Psi_{e}(t)\right\rangle= & \frac{1}{6}\left|\psi_{\mathrm{IV}, 0, e}\right\rangle-\frac{1}{12} \sum_{\sigma= \pm} e^{-i \sigma \sqrt{6} J t}\left(\left|\psi_{\mathrm{II}, \sigma, c}\right\rangle\right. \\
& \left.+\left|\psi_{\mathrm{I}, \sigma, d_{l}}\right\rangle+\left|\psi_{\mathrm{I}, \sigma, d_{r}}\right\rangle\right) \\
= & \frac{1}{6}[\beta-1, \beta-1, \beta-1,0,-\sqrt{6} \alpha, \beta-1, \beta-1 \\
& \quad-\sqrt{6} \alpha, 3(\beta+1), 0,1-\beta, 0,-\sqrt{6} \alpha \\
& \quad \beta-1, \beta-1,1-\beta]^{T} .
\end{aligned}
$$

The fidelity as the overlap between the initial state and the time-evolution state is

$$
F_{e}(t)=\left|\left\langle\Psi_{e}(0) \mid \Psi_{e}(t)\right\rangle\right|=\frac{1}{2}(\beta+1) .
$$

For the single-site excitation at site $f$ as shown in Fig. 5(f), initial-state $\left|\Psi_{f}(0)\right\rangle$ is a superposition of seven CLSs with a type-VI CLS centered at site $f$ and three pairs of nonzero-energy CLSs localized at the snowflake centers. The 
time-evolution state $\left|\Psi_{f}(t)\right\rangle$ is

$$
\begin{aligned}
\left|\Psi_{f}(t)\right\rangle= & \frac{1}{6}\left|\psi_{\mathrm{VI}, 0, f}\right\rangle+\frac{1}{12} \sum_{\sigma= \pm} e^{-i \sigma \sqrt{6} J t}\left(\left|\psi_{\mathrm{I}, \sigma, d_{l}}\right\rangle\right. \\
& \left.-\left|\psi_{\mathrm{I}, \sigma, d_{r}}\right\rangle-\left|\psi_{\mathrm{II}, \sigma, c}\right\rangle\right) \\
= & \frac{1}{6}[1-\beta, 1-\beta, 1-\beta, \sqrt{6} \alpha, 0,1-\beta, 0, \\
& \quad 3(\beta+1),-\sqrt{6} \alpha, \beta-1, \beta-1,-\sqrt{6} \alpha, \\
& 0,1-\beta, \beta-1,1-\beta]^{T} .
\end{aligned}
$$

Figure $6(\mathrm{~b})$ presents the numerical simulation of the time evolution for a single-site excitation on a branch site of the snowflake $\left|\Psi_{f}(t)\right\rangle$. The fidelity as the overlap between the initial state and the time-evolution state is

$$
F_{f}(t)=\left|\left\langle\Psi_{f}(0) \mid \Psi_{f}(t)\right\rangle\right|=\frac{1}{2}(\beta+1) .
$$

Figure 6 shows the dynamics of the typical situations for the single-site initial excitation. The time-evolution dynamics is periodic for the excitation, and the period is $2 \pi /(\sqrt{6} J) \approx$ $2.57 J^{-1}$ obtained from the nonzero flatband energy. The fidelity of the time-evolved excitation $F(t)=|\langle\Psi(0) \mid \Psi(t)\rangle|$ is shown at the left side of the plots.

It is worth mentioning that the two localization dynamics elaborated in Fig. 6 are the only two classes of localization behaviors. Generally, Fig. 6(a) represents the localization dynamics for a single-site excitation at the center site of the snowflake; and Fig. 6(b) represents the localization dynamics for a single-site excitation at the branch site of the snowflake. With all the analytical results of single-site excitation for the six types of sublattices, any initial state is a superposition of these six types of dynamics.

In Fig. 7(a), we perform the numerical simulations for the initial excitation being a snowflake with all seven sites equally excited at the same strength, and the excitation amplitudes on different sites have the same phase. As an example of the general excitation, we show that the localization of a general excitation can be roughly grasped from the two typical dynamical behaviors of a single-site excitation. The fidelity obtained from the time evolution dynamics is

$$
F(t)=\frac{1}{21}|11 \beta+10| \text {. }
$$

In Fig. 7(b), the numerical simulation is performed for the initial excitation on the four centers of four nearest hexagonal structures that are coupled in the configuration of a trident star; the four sites are equally excited at the same strength and the same phase. In both situations shown in the first two rows of Fig. 7, the excitations are completely confined within the seven hexagonal structures close to the excitations. The fidelity obtained from the time-evolution dynamics is

$$
F(t)=\frac{1}{24}|13 \beta+11| .
$$

In Fig. 7(c), we perform the numerical simulations for the initial excitation that equally excites the four sites of a diamond plaquette at the same strength and the same phase; the coefficients of the excitation on the diamond plaquette are 1:1:1:1. Note that among the four sites of the diamond plaquette, two of them are the snowflake centers, and the other two sites are the branch sites of two other snowflakes; thus, the diamond plaquette is relevant to four nearest-neighbor snowflakes shown in Fig. 7(c) and the excitation is always confined in the relevant four snowflakes in the whole timeevolution process. The analytical expression is given in the Appendix from which we obtain the fidelity,

$$
F(t)=\frac{1}{4}|3 \beta+1| .
$$

In Fig. 7(d), we perform the numerical simulations for the initial excitation that equally excites three sites on a vertical line, the coefficients of the excitation on the three sites are 1:1:1. Note that among the three excited sites, one site (on the top) is the snowflake center, and the other two sites (in the middle and at the bottom) are branch sites. Each branch site is relevant to three snowflakes, but the two different branch sites being excited share two of the relevant snowflakes; and the top site share the snowflake where it centers. Thus, the excitation is confined in four nearest-neighbor snowflakes shown in Fig. 7(d) in the whole time-evolution process. The analytical expression is given in the Appendix from which we obtain the fidelity,

$$
F(t)=\frac{1}{3}|2 \beta+1| .
$$

Due to the flatness of all energy levels, such dice lattice we discussed present localization when performing the time evolution. Any initial state is confined in a certain area that is related to CLS configurations. Dynamics only for CLSs is nondiffractive, and the intensity distribution is constant for each site. From all the situations that are exhibited in Figs. 6 and 7, we note that the localization dynamics of an initial excitation in the general case is compactly localized in the snowflakes that relates to the sites being excited as our expectation and the period of the time evolution is $2 \pi /(\sqrt{6} J)$.

The quench dynamics in the $\alpha-\mathcal{T}_{3}$ lattice and the dynamical quantum phase transition are recently reported through investigating the three-band Bloch Hamiltonian in the momentum space [74]. The quench dynamics is a powerful approach in extracting the properties of the physical system. The quench dynamics in the dice lattice enclosed with tunable magnetic flux is an interesting topic for future study. Alternatively, the capability of calculating the dynamics of arbitrary excitation enables us to investigate the quench dynamics in the real space by taking the dice lattice Hamiltonian as the finial Hamiltonian after quench. The confinement dynamics can be understood from a different viewpoint.

\section{CONCLUSION}

The dice $\left(\mathcal{T}_{3}\right)$ lattice supports $\mathrm{AB}$ caging when the halfquantum magnetic flux is enclosed in the diamond plaquette of the dice lattice. In this situation, the dice lattice has a six-site unit cell; and all the six energy bands form three doubly degenerate flatbands, being symmetric about the zero energy. We have analytically presented the eigenstates of the dice lattice, which are the CLSs that are localized in the snowflake structures of the dice lattice. The CLSs of every flatband has two inequivalent configurations of wavefunction distributions according to the double degeneracy of the flatband. The CLSs of the nonzero-energy flatbands 

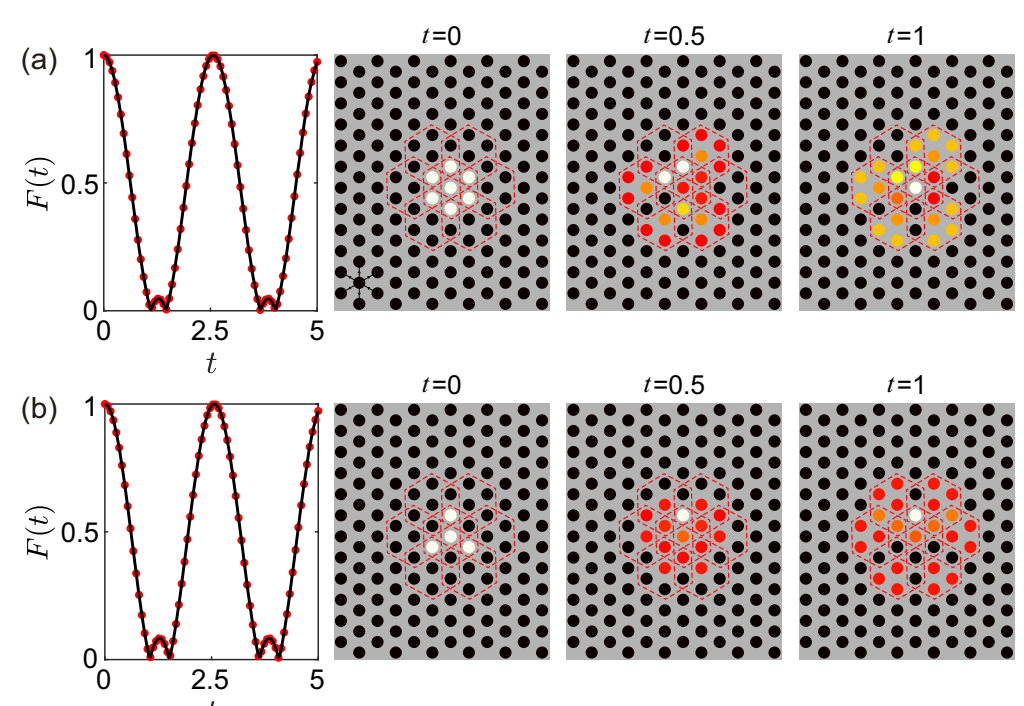

$t=0.5$
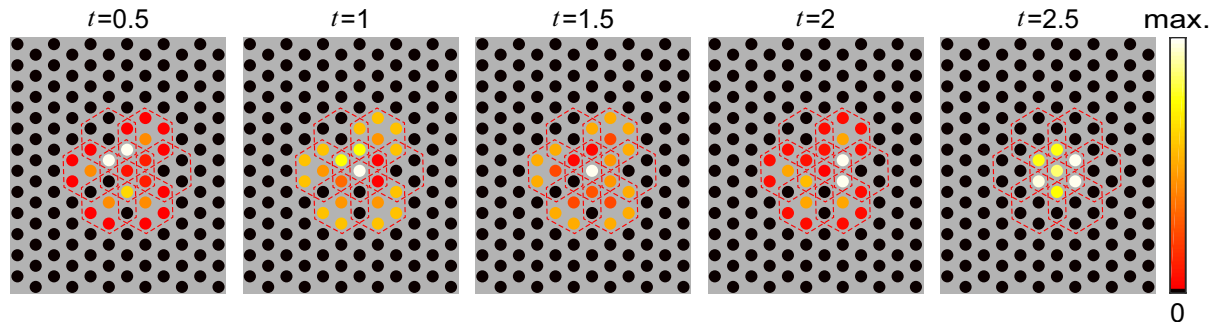

$t=1.5$
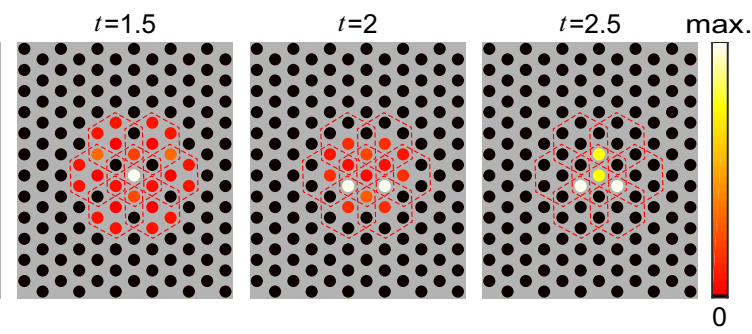

$t=1.5$
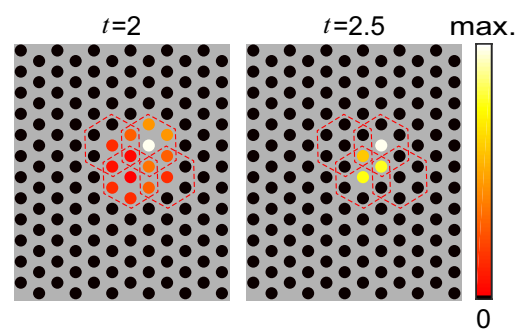

0
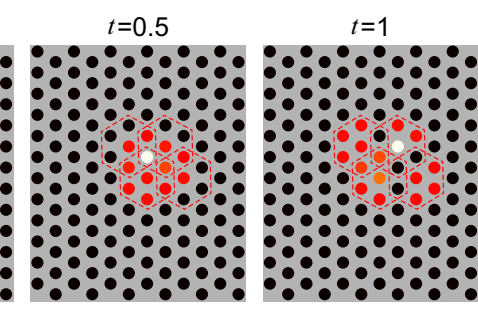

$t=1$

$t=1.5$
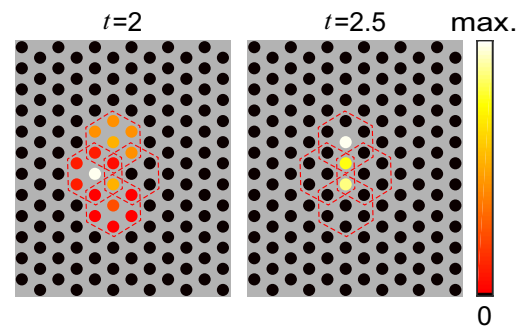

FIG. 7. Fidelity $F(t)=|\langle\Psi(0) \mid \Psi(t)\rangle|$ and snapshots of the time-evolved excitation intensity $\langle\Psi(t) \mid \Psi(t)\rangle$ for the initial excitation $|\Psi(0)\rangle$ of (a) a snowflake with all seven sites; (b) a trident star with all four sites; (c) a diamond plaquette with all four sites; and (d) a vertical line of three neighbor sites. The initial state is normalized, $\langle\Psi(0) \mid \Psi(0)\rangle=1$. The analytical results of $F(t)$ are indicated by the black solid lines; the numerical results of $F(t)$ are in the red dots. In all the cases, the excitation has equal amplitude and same phase on each site. The dice lattice has 144 sites and the coupling strength is $J=1$. The lattice couplings are indicated in the left bottom corner of the snapshots of the four cases at $t=0$. The numbers in (c) and (d) label the index for the wave functions of $\Psi(t)$ given in the Appendix.

are localized in a single snowflake; all the nonzero-energy CLSs are linearly independent. In contrast, the CLSs of the zero-energy flatband are localized in three nearest-neighbor snowflakes that are arranged in the form of a trident star; there are four types of zero-energy CLSs configurations, but only part of them supported in the dice lattice are linearly independent. The single-site initial excitations are either confined in a single snowflake or confined in the relevant three connected snowflakes. The localization dynamics of the $\mathrm{AB}$ caging is in accord with the analytical results for the CLSs of the dice lattice. In general, arbitrary initial excitation is compactly confined within the relevant snowflakes that relates to the excited sites. Moreover, the oscillation period of the $\mathrm{AB}$ caging is $2 \pi /(\sqrt{6} J)$. In the future, it would be interesting to further investigate the influences of nonlinearity and non-
Hermiticity on the spectrum and localization dynamics in the dice lattice $[10,11,45]$.

\section{ACKNOWLEDGMENT}

We acknowledge the support from National Natural Science Foundation of China under Grant No. 11975128.

\section{APPENDIX}

\section{Orthogonality of degenerate CLSs}

The CLSs from different flatbands have different energies and are orthogonal to each other. Thus, we focus on the orthogonality of the degenerate CLSs. We consider the degenerate CLSs of type-I and type-II. The two CLSs of type-I 
(a)
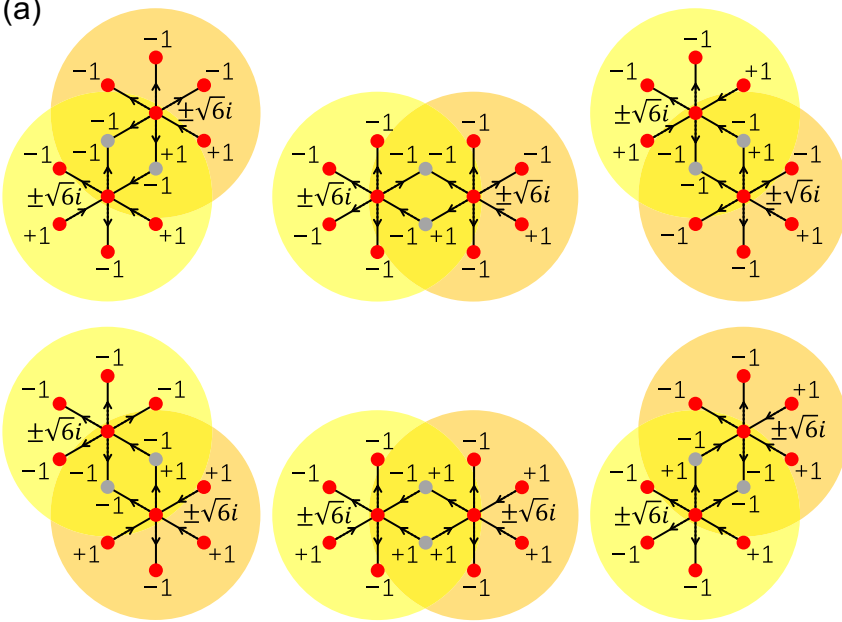

(b)

(c)
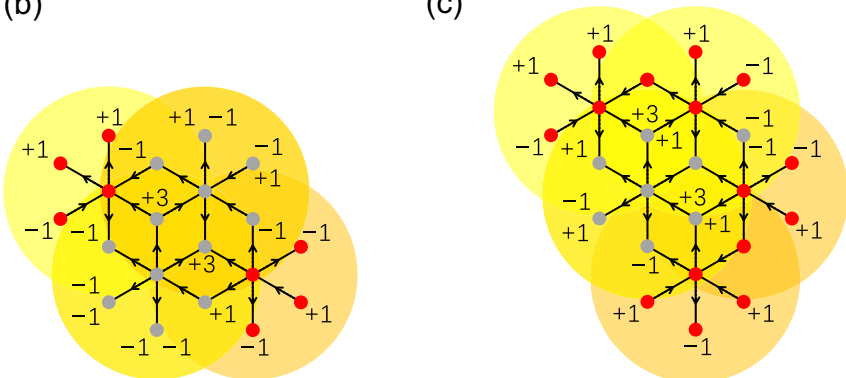

FIG. 8. (a) Schematic of the orthogonality for CLSs of type-I and type-II that are localized in the nearest-neighbor snowflakes. All the situations are exemplified. (b) Orthogonal CLSs with zero overlap between CLSs of type-III and type-IV. (c) Nonorthogonal CLSs with nonzero overlap between CLSs of type-III and type-VI. The wave-function values of CLSs are marked. The shared sites of the two CLSs are shaded in gray.

or two CLSs of type-II may present in two nearest-neighbor snowflakes in the horizontal direction; alternatively, the two CLSs with one type-I and one type-II can localize nearby below or above each other. In all the situations, the CLSs of type-I and type-II localized nearby share two sites of the snowflakes as exhibited in Fig. 8(a). The overlap between different CLSs of type-I and type-II are directly calculated from the marked wave-function values, that is $1+(-1)=0$ for either two CLSs shaded in different colors. Moreover, the degenerate CLSs of type-I and type-II are still orthogonal to each other. To summarize, all the CLSs of type-I and type-II are orthogonal and linearly independent, regardless of their energies.

The eigenstates of the zero-energy flatband are CLSs from type-III to type-VI. In the dice lattice of Fig. 1, both the first and the third horizontal lines from the top of the dice lattice with four snowflakes support four type-III and four type-V zero-energy CLSs; both the second and the fourth horizontal lines from the top of the dice lattice with four snowflakes support four type-IV and four type-VI zero-energy CLSs. There are, in total, 32 CLSs of type-III to type-VI; however, all the CLSs of type-III to type-VI are not fully linearly independent, and only part of them are linearly independent.
The fact is noted from the zero and nonzero overlaps between the CLSs of type-III to type-VI. For example, the overlap between the CLSs of type-III and type-IV shown in Fig. 8(b) is zero; similarly, the overlap between the CLSs of type-V and type-VI as the nearest neighbors is also zero.

In contrast, two CLSs of type-III and type-VI localized vertically as the nearest neighbor share eight sites as shown in Fig. 8(c), their overlap is nonzero; moreover, one CLS of type-III and one CLS of type-V localized vertically as the nearest neighbor share seven sites, their overlap is also nonzero.

\section{Examples of the time-evolution dynamics}

We elucidate how to obtain the dynamics of an arbitrary excitation from the dynamics of single-site excitations presented in Sec. IV. Two examples in Fig. 7 are elucidated as follows.

In Fig. 7(c), initial-state $|\Psi(0)\rangle$ with sites 8, 9, 12, and 13 equally excited is an equal amplitude superposition of four single-site excitations shown in Eqs. (33), (27), (29), and (23). The superposition coefficients are $1 / 2 .\left|\Psi_{8}(t)\right\rangle$, $\left|\Psi_{9}(t)\right\rangle,\left|\Psi_{12}(t)\right\rangle$, and $\left|\Psi_{13}(t)\right\rangle$ represent the time evolution of the single-site excitation at $8,9,12$, and 13 , respectively. The time-evolution state $|\Psi(t)\rangle$ is

$$
\begin{aligned}
|\Psi(t)\rangle=\frac{1}{2}[ & \left.\left|\Psi_{8}(t)\right\rangle+\left|\Psi_{9}(t)\right\rangle+\left|\Psi_{12}(t)\right\rangle+\left|\Psi_{13}(t)\right\rangle\right] \\
=\frac{1}{12} & {[1-\beta, 1-\beta, 1-\beta, \sqrt{6} \alpha, \sqrt{6} \alpha-\beta+1,} \\
& -\sqrt{6} \alpha, \beta+\sqrt{6} \alpha-1,3 \beta+2 \sqrt{6} \alpha+3,6 \beta, \\
& \sqrt{6} \alpha, 2 \beta+\sqrt{6} \alpha-2,6 \beta-2 \sqrt{6} \alpha, 3(\beta+1), \\
& 1-\sqrt{6} \alpha-\beta, 2 \beta+\sqrt{6} \alpha-2,1-\sqrt{6} \alpha-\beta, \\
& -\sqrt{6} \alpha, \beta-1,1-\beta, 1-\beta]^{T},
\end{aligned}
$$

where the numbers in Fig. 7(c) label the index for the wave functions of $|\Psi(t)\rangle$; the amplitude on the other sites without labels are all zeros. The fidelity in Eq. (37) is obtained from $|\Psi(t)\rangle$ in Eq. (A1).

In Fig. 7(d), initial state $|\Psi(0)\rangle$ with sites 9-11 equally excited is an equal amplitude superposition of three single-site excitations shown in Eqs. (29), (23), and (25). The superposition coefficients are $\sqrt{3} / 3 .\left|\Psi_{9}(t)\right\rangle,\left|\Psi_{10}(t)\right\rangle$, and $\left|\Psi_{11}(t)\right\rangle$ represent the time evolution of the single-site excitation at 9-11, respectively. The time-evolution state $|\Psi(t)\rangle$ is

$$
\begin{aligned}
|\Psi(t)\rangle= & \frac{\sqrt{3}}{3}\left[\left|\Psi_{9}(t)\right\rangle+\left|\Psi_{10}(t)\right\rangle+\left|\Psi_{11}(t)\right\rangle\right] \\
= & \frac{\sqrt{3}}{18}[2(1-\beta), 2(\beta-1), \beta+\sqrt{6} \alpha-1, \\
& \sqrt{6} \alpha-\beta+1,2 \sqrt{6} \alpha, 1-\beta, \beta-1, \beta+\sqrt{6} \alpha-1, \\
& 6 \beta-\sqrt{6} \alpha, 3 \beta+\sqrt{6} \alpha+3,3(\beta+1),-\sqrt{6} \alpha, \\
& \beta-1, \beta+\sqrt{6} \alpha-1,1-\sqrt{6} \alpha-\beta, 0, \\
& \beta-1,1-\beta, 0,0]^{T},
\end{aligned}
$$

where the numbers in Fig. 7(d) label the index for the wave functions of $|\Psi(t)\rangle$; the amplitude on the other sites without labels are all zeros. The fidelity in Eq. (38) is obtained from $|\Psi(t)\rangle$ in Eq. (A2). 
[1] D. Leykam, A. Andreanov, and S. Flach, Artificial flat band systems: From lattice models to experiments, Adv. Phys. X 3, 1473052 (2018).

[2] S. Flach, D. Leykam, J. D. Bodyfelt, P. Matthies, and A. S. Desyatnikov, Detangling flat bands into Fano lattices, Europhys. Lett. 105, 30001 (2014).

[3] J. Vidal, B. Doucot, R. Mosseri, and P. Butaud, Interaction Induced Delocalization for Two Particles in a Periodic Potential, Phys. Rev. Lett. 85, 3906 (2000).

[4] C. E. Creffield and G. Platero, Coherent Control of Interacting Particles Using Dynamical and Aharonov-Bohm Phases, Phys. Rev. Lett. 105, 086804 (2010).

[5] B. Douçot and J. Vidal, Pairing of Cooper Pairs in a Fully Frustrated Josephson-Junction Chain, Phys. Rev. Lett. 88, 227005 (2002).

[6] A. Bermudez, T. Schaetz, and D. Porras, Synthetic Gauge Fields for Vibrational Excitations of Trapped Ions, Phys. Rev. Lett. 107, 150501 (2011).

[7] D. Leykam, O. Bahat-Treidel, and A. S. Desyatnikov, Flat band states: Disorder and nonlinearity, Phys. Rev. B 88, 224203 (2013).

[8] S. Mukherjee and R. R. Thomson, Observation of localized flat-band modes in a quasi-one-dimensional photonic rhombic lattice, Opt. Lett. 40, 5443 (2015).

[9] S. Mukherjee and R. R. Thomson, Observation of robust flatband localization in driven photonic rhombic lattices, Opt. Lett. 42, 2243 (2017).

[10] G. Gligorić, P. P. Beličev, D. Leykam, and A. Maluckov, Nonlinear symmetry breaking of Aharonov-Bohm cages, Phys. Rev. A 99, 013826 (2019).

[11] M. D. Liberto, S. Mukherjee, and N. Goldman, Nonlinear dynamics of Aharonov-Bohm cages, Phys. Rev. A 100, 043829 (2019).

[12] R. Khomeriki and S. Flach, Landau-Zener Bloch Oscillations with Perturbed Flat Bands, Phys. Rev. Lett. 116, 245301 (2016).

[13] L. Morales-Inostroza and R. A. Vicencio, Simple method to construct flat-band lattices, Phys. Rev. A 94, 043831 (2016).

[14] W. Maimaiti, A. Andreanov, H. C. Park, O. Gendelman, and S. Flach, Compact localized states and flat-band generators in one dimension, Phys. Rev. B 95, 115135 (2017).

[15] J. D. Bodyfelt, D. Leykam, C. Danieli, X. Yu, and S. Flach, Flatbands under Correlated Perturbations, Phys. Rev. Lett. 113, 236403 (2014).

[16] M. Tovmasyan, S. Peotta, L. Liang, P. Törmä, and S. D. Huber, Preformed pairs in flat Bloch bands, Phys. Rev. B 98, 134513 (2018).

[17] C. Gneiting, Z. Li, and F. Nori, Lifetime of flatband states, Phys. Rev. B 98, 134203 (2018).

[18] J. Vidal, R. Mosseri, and B. Douçot, Aharonov-Bohm Cages in Two-Dimensional Structures, Phys. Rev. Lett. 81, 5888 (1998).

[19] J. Vidal, P. Butaud, B. Douçot, and R. Mosseri, Disorder and interactions in Aharonov-Bohm cages, Phys. Rev. B 64, 155306 (2001).

[20] C. Naud, G. Faini, and D. Mailly, Aharonov-Bohm Cages in 2D Normal Metal Networks, Phys. Rev. Lett. 86, 5104 (2001).

[21] A. R. Kolovsky, A. Ramachandran, and S. Flach, Topological flat Wannier-Stark bands, Phys. Rev. B 97, 045120 (2018).

[22] C. Wu, D. Bergman, L. Balents, and S. Das Sarma, Flat Bands and Wigner Crystallization in the Honeycomb Optical Lattice, Phys. Rev. Lett. 99, 070401 (2007).
[23] T. Jacqmin, I. Carusotto, I. Sagnes, M. Abbarchi, D. D. Solnyshkov, G. Malpuech, E. Galopin, A. Lemaître, J. Bloch, and A. Amo, Direct Observation of Dirac Cones and a Flatband in a Honeycomb Lattice for Polaritons, Phys. Rev. Lett. 112, 116402 (2014).

[24] N. Masumoto, N. Y. Kim, T. Byrnes, K. Kusudo, A. Löffler, S. Höfling, A. Forchel, and Y. Yamamoto, Exciton-polariton condensates with flat bands in a two-dimensional kagome lattice, New J. Phys. 14, 065002 (2012).

[25] G.-W. Chern, C.-C. Chien, and M. Di Ventra, Dynamically generated flat-band phases in optical kagome lattices, Phys. Rev. A 90, 013609 (2014).

[26] G.-B. Jo, J. Guzman, C. K. Thomas, P. Hosur, A. Vishwanath, and D. M. Stamper-Kurn, Ultracold Atoms in a Tunable Optical Kagome Lattice, Phys. Rev. Lett. 108, 045305 (2012).

[27] J. T. Chalker, T. S. Pickles, and P. Shukla, Anderson localization in tight-binding models with flat bands, Phys. Rev. B 82, 104209 (2010).

[28] P. Shukla, Disorder perturbed flat bands: Level density and inverse participation ratio, Phys. Rev. B 98, 054206 (2018).

[29] T. Mizoguchi and M. Udagawa, Flat-band engineering in tight-binding models: Beyond the nearest-neighbor hopping, Phys. Rev. B 99, 235118 (2019).

[30] E. H. Lieb, Two Theorems on the Hubbard Model, Phys. Rev. Lett. 62, 1201 (1989).

[31] F. Baboux, L. Ge, T. Jacqmin, M. Biondi, E. Galopin, A. Lemaître, L. Le Gratiet, I. Sagnes, S. Schmidt, H. E. Türeci, A. Amo, and J. Bloch, Bosonic Condensation and DisorderInduced Localization in a Flat Band, Phys. Rev. Lett. 116 066402 (2016).

[32] L. Ge, Anomalous minimum and scaling behavior of localization length near an isolated flat band, Ann. Phys. (Berlin) 529, 1600182 (2017).

[33] A. Julku, S. Peotta, T. I. Vanhala, D.-H. Kim, and P. Törmä, Geometric Origin of Superfluidity in the Lieb-Lattice Flat Band, Phys. Rev. Lett. 117, 045303 (2016).

[34] D. Guzmán-Silva, C. Mejía-Cortés, M. A. Bandres, M. C. Rechtsman, S. Weimann, S. Nolte, M. Segev, A. Szameit, and R. A. Vicencio, Experimental observation of bulk and edge transport in photonic Lieb lattices, New J. Phys. 16, 063061 (2014).

[35] R. A. Vicencio, C. Cantillano, L. Morales-Inostroza, B. Real, C. Mejía-Cortés, S. Weimann, A. Szameit, and M. I. Molina, Observation of Localized States in Lieb Photonic Lattices, Phys. Rev. Lett. 114, 245503 (2015).

[36] S. Mukherjee, A. Spracklen, D. Choudhury, N. Goldman, P. Öhberg, E. Andersson, and R. R. Thomson, Observation of a Localized Flat-Band State in a Photonic Lieb Lattice, Phys. Rev. Lett. 114, 245504 (2015).

[37] V. Apaja, M. Hyrkás, and M. Manninen, Flat bands, Dirac cones, and atom dynamics in an optical lattice, Phys. Rev. A 82, 041402 (2010).

[38] H. Ozawa, S. Taie, T. Ichinose, and Y. Takahashi, InteractionDriven Shift and Distortion of a Flat Band in an Optical Lieb Lattice, Phys. Rev. Lett. 118, 175301 (2017).

[39] M. R. Slot, T. S. Gardenier, P. H. Jacobse, G. C. P. van Miert, S. N. Kempkes, S. J. M. Zevenhuizen, C. M. Smith, D. Vanmaekelbergh, and I. Swart, Experimental realization and characterization of an electronic Lieb lattice, Nat. Phys. 13, 672 (2017). 
[40] T. Biesenthal, M. Kremer, M. Heinrich, and A. Szameit, Experimental Realization of $\mathcal{P} \mathcal{T}$-Symmetric Flat Bands, Phys. Rev. Lett. 123, 183601 (2019).

[41] M. I. Molina, Flat bands and $\mathcal{P} \mathcal{T}$ symmetry in quasi-onedimensional lattices, Phys. Rev. A 92, 063813 (2015).

[42] L. Ge, Parity-time symmetry in a flat-band system, Phys. Rev. A 92, 052103 (2015).

[43] H. Ramezani, Non-Hermiticity-induced flat band, Phys. Rev. A 96, 011802(R) (2017).

[44] D. Leykam, S. Flach, and Y. D. Chong, Flat bands in lattices with non-Hermitian coupling, Phys. Rev. B 96, 064305 (2017).

[45] L. Ge, Non-Hermitian lattices with a flat band and polynomial power increase, Photon. Res. 6, A10 (2018).

[46] B. Qi, L. Zhang, and L. Ge, Defect States Emerging from a Non-Hermitian Flatband of Photonic Zero Modes, Phys. Rev. Lett. 120, 093901 (2018).

[47] L. Jin, Flat band induced by the interplay of synthetic magnetic flux and non-Hermiticity, Phys. Rev. A 99, 033810 (2019).

[48] A. Ramachandran, A. Andreanov, and S. Flach, Chiral flat bands: Existence, engineering, and stability, Phys. Rev. B 96, 161104(R) (2017).

[49] S. Longhi, Aharonov-Bohm photonic cages in waveguide and coupled resonator lattices by synthetic magnetic fields, Opt. Lett. 39, 5892 (2014).

[50] S. Mukherjee, M. Di Liberto, P. Öhberg, R. R. Thomson, and N. Goldman, Experimental Observation of Aharonov-Bohm Cages in Photonic Lattices, Phys. Rev. Lett. 121, 075502 (2018).

[51] M. Kremer, I. Petrides, E. Meyer, M. Heinrich, O. Zilberberg, and A. Szameit, A square-root topological insulator with non-quantized indices realized with photonic Aharonov-Bohm cages, Nat. Commun. 11, 907 (2020).

[52] S. E. Korshunov, Vortex ordering in fully frustrated superconducting systems with a dice lattice, Phys. Rev. B 63, 134503 (2001).

[53] D. Bercioux, D. F. Urban, H. Grabert, and W. Häusler, Massless Dirac-Weyl fermions in a $\mathcal{T}_{3}$ optical lattice, Phys. Rev. A $\mathbf{8 0}$, 063603 (2009).

[54] J. D. Malcolm and E. J. Nicol, Frequency-dependent polarizability, plasmons, and screening in the two-dimensional pseudospin-1 dice lattice, Phys. Rev. B 93, 165433 (2016).

[55] G. Möller and N. R. Cooper, Correlated Phases of Bosons in the Flat Lowest Band of the Dice Lattice, Phys. Rev. Lett. 108, 045306 (2012).

[56] F. Wang and Y. Ran, Nearly flat band with Chern number $C=2$ on the dice lattice, Phys. Rev. B 84, 241103 (2011).

[57] T. Andrijauskas, E. Anisimovas, M. Račiūnas, A. Mekys, V. Kudriašov, I. B. Spielman, and G. Juzeliūnas, Three-level Haldane-like model on a dice optical lattice, Phys. Rev. A 92 , 033617 (2015).
[58] G. Möller and N. R. Cooper, Synthetic gauge fields for lattices with multi-orbital unit cells: Routes towards a $\pi$-flux dice lattice with flat bands, New J. Phys. 20, 073025 (2018).

[59] R. Soni, N. Kaushal, S. Okamoto, and E. Dagotto, Flat bands and ferrimagnetic order in electronically correlated dice-lattice ribbons, Phys. Rev. B 102, 045105 (2020).

[60] M. Rizzi, V. Cataudella, and R. Fazio, Phase diagram of the Bose-Hubbard model with $\mathcal{T}_{3}$ symmetry, Phys. Rev. B 73, 144511 (2006).

[61] M. Payrits and R. Barnett, Order-by-disorder degeneracy lifting of interacting bosons on the dice lattice, Phys. Rev. A 90, 013608 (2014).

[62] E. Illes, J. P. Carbotte, and E. J. Nicol, Hall quantization and optical conductivity evolution with variable Berry phase in the $\alpha-\mathcal{T}_{3}$ model, Phys. Rev. B 92, 245410 (2015).

[63] Á. D. Kovács, G. Dávid, B. Dóra, and J. Cserti, Frequencydependent magneto-optical conductivity in the generalized $\alpha-\mathcal{T}_{3}$ model, Phys. Rev. B 95, 035414 (2017).

[64] S. K. Firoz Islam and P. Dutta, Valley-polarized magnetoconductivity and particle-hole symmetry breaking in a periodically modulated $\alpha-\mathcal{T}_{3}$ lattice, Phys. Rev. B 96, 045418 (2017).

[65] Y.-R. Chen, Y. Xu, J. Wang, J.-F. Liu, and Z. Ma, Enhanced magneto-optical response due to the flat band in nanoribbons made from the $\alpha-\mathcal{T}_{3}$ lattice, Phys. Rev. B 99, 045420 (2019).

[66] E. V. Gorbar, V. P. Gusynin, and D. O. Oriekhov, Electron states for gapped pseudospin-1 fermions in the field of a charged impurity, Phys. Rev. B 99, 155124 (2019).

[67] B. Dey and T. K. Ghosh, Floquet topological phase transition in the $\alpha-\mathcal{T}_{3}$ lattice, Phys. Rev. B 99, 205429 (2019).

[68] A. A. Burkov and E. Demler, Vortex-Peierls States in Optical Lattices, Phys. Rev. Lett. 96, 180406 (2006).

[69] M. Hafezi, S. Mittal, J. Fan, A. Migdall, and J. M. Taylor, Imaging topological edge states in silicon photonics, Nat. Photon. 7, 1001 (2013).

[70] S. Mittal, S. Ganeshan, J. Fan, A. Vaezi, and M. Hafezi, Measurement of topological invariants in a 2D photonic system, Nat. Photon. 10, 180 (2016).

[71] D. Leykam, K. Y. Bliokh, C. Huang, Y. D. Chong, and F. Nori, Edge Modes, Degeneracies, and Topological Numbers in NonHermitian Systems, Phys. Rev. Lett. 118, 040401 (2017).

[72] D. Leykam, S. Mittal, M. Hafezi, and Y. D. Chong, Reconfigurable Topological Phases in Next-Nearest-Neighbor Coupled Resonator Lattices, Phys. Rev. Lett. 121, 023901 (2018).

[73] L. Jin and Z. Song, Incident Direction Independent Wave Propagation and Unidirectional Lasing, Phys. Rev. Lett. 121, 073901 (2018).

[74] B. Gulácsi, M. Heyl, and B. Dóra, Geometrical quench and dynamical quantum phase transition in the $\alpha-T_{3}$ lattice, Phys. Rev. B 101, 205135 (2020). 